\title{
Tantalizing tantalum
}

Giovanni Baccolo relates tales of tantalum, an element known, and named, for its inertness, yet one that holds some surprises, such as a naturally occurring nuclear isomer.

Element 73 was first extracted from mineral samples and described in 1802 by the Swedish chemist Anders Ekeberg. He chose the name tantalum because "when placed in the midst of acids it is incapable of taking any of them up and saturating itself with them", in a manner reminiscent of the Greek mythological king Tantalus (pictured) who couldn't eat or drink anything despite being surrounded by food and water ${ }^{1}$.

For over six decades tantalum and its neighbour in the periodic table, niobium - discovered in 1801 by Charles Hatchett and initially named columbium - were thought to be one and the same. They share very similar chemical and physical properties, and are always found together in nature. The discoveries of columbium, tantalum, pelopium, niobium and ilmenium were all repeatedly claimed between 1801 and 1866 , however, these proved to be just tantalum, niobium and mixtures of the two.

It was the French scientist Jean Charles Galissard de Marignac who first succeeded in separating tantalum from niobium, by exploiting the different solubilities of their fluorinated compounds ${ }^{2}$. Tantalum belongs to the refractory metals along with - perhaps unsurprisingly - niobium, as well as molybdenum, tungsten and rhenium. These elements have extremely elevated melting points (tantalum melts at 3,290 K), and notoriously high mechanical and chemical resistance.

This inertness is what makes tantalum useful for a variety of practical purposes. Its first applications date from the beginning of the twentieth century, on the heels of new extraction and purification methods. In 1905 engineers employed by Ernst Werner von Siemens designed an incandescent light bulb based on a tantalum filament, in the first commercial attempt to replace fragile carbon filaments with a metal. Tantalum bulbs however were soon replaced by another refractory metal,

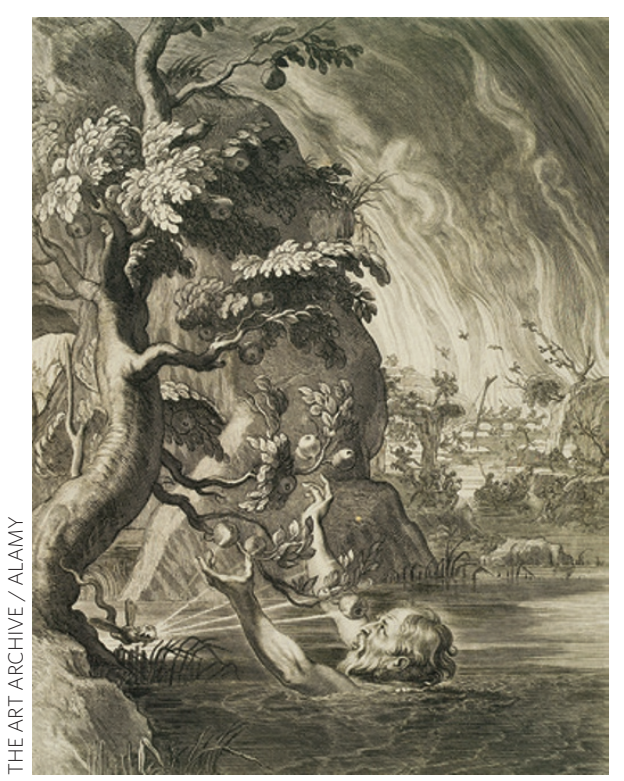

tungsten, more suitable because it is less volatile and has an even higher melting point $(3,695 \mathrm{~K})$. Tantalum has also been used to make fountain pen nibs that were very resistant to ink, but was soon replaced with osmium- and iridium-based alloys. Modern applications of tantalum span from the production of alloys, to electronics - mobile phones and similar devices often rely on tantalum capacitors - to surgical implants, where inertness ensures that it doesn't interfere with biological tissues or processes.

The most peculiar features of element 73 involve some of its nuclear properties. Tantalum was initially believed to have only one natural isotope, ${ }^{181} \mathrm{Ta}$, but in 1955 a second was identified ${ }^{3},{ }^{180} \mathrm{Ta}$. Its relative abundance is estimated to be only $0.01201 \%$ of natural tantalum, making it the rarest naturally occurring isotope observed so far. The half-life of ${ }^{180} \mathrm{Ta}$ is about 8 hours, which means that it should be radioactive - so how is it possible to observe it in natural samples?

This isotope exists as ${ }^{180 \mathrm{~m}} \mathrm{Ta}$, in which the ' $m$ ' denotes a metastable state, called isomer in nuclear physics. An isomer thus lies in the excited state of an atomic nucleus, in the same manner as excited electrons do. They are typically radioactive species that decay quickly to the ground state emitting characteristic gamma rays. But sometimes they take longer times to decay. This is the case with ${ }^{180 \mathrm{~m}} \mathrm{Ta}$, which is the most stable and the only naturally occurring nuclear isomer known. Despite several attempts, its spontaneous decay hasn't been observed yet; only lower limits of half-life have been posed, in the range of $10^{12}-10^{16}$ years. Astrophysicists tried to shed some light on the origin of this peculiar isotope by investigating the classical nucleosynthesis pathways occurring in the stars, as well as extreme events such as supernova explosions, nuclear captures and disintegrations - but a consensus has yet to be reached. Understanding this elusive process will be an important test for nucleosynthesis models.

Another important aspect of ${ }^{180 \mathrm{~m} T a}$, and the other long-lived nuclear isomers, is that it may be possible to force their decay to the ground state, inducing the release of energy in the form of gamma ray emission. Some successful attempts have already been carried out with ${ }^{180 \mathrm{~m}} \mathrm{Ta}$, but the process is far from energetically efficient ${ }^{4}$. Improving this process may lead to the development of gamma ray lasers, or perhaps a new kind of nuclear battery ${ }^{5}$.

It may be better known for its inertness and chemical and mechanical resistance, but element 73 also poses intriguing questions.

\section{GIOVANNI BACCOLO is in the Earth}

Science Department, University of Siena, via Laterina 8, 53100, Siena, Italy. e-mail: giovanni.baccolo@mib.infn.it

\section{References}

1. Ekeberg, A. G. J. Nat. Philos. Chem. Arts 3, 251-255 (1802).

2. Marignac, M. C. Ann. Chim. Phys. 4, 7-5 (1866).

3. White, F. A., Collins, T. L. Jr \& Rourke, F. M. Phys. Rev. 97, 566-567 (1955).

4. Collins, C. B., Eberhard, C. D., Glesener, J. W. \& Anderson, J. A. Phys. Rev. C 37, 2267-2269 (1988).

5. Walker, P. \& Dracoulis, G. Nature 399, 35-40 (1997)
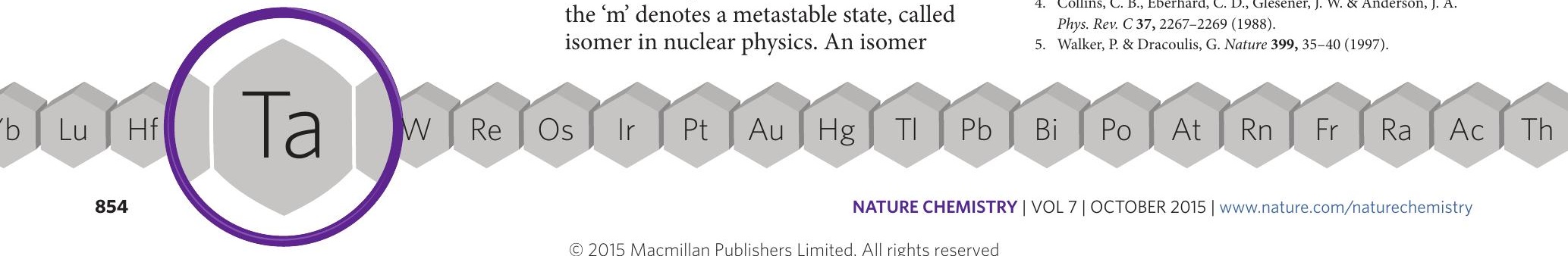